\title{
Correlation between diffraction and viscosity data for Bi-Ga molten alloys
}

\author{
S MUDRY, A YAKYMOVYCH*, I SHTABLAVYI, V SIDOROV ${ }^{\dagger}$ and K SHUNYAEV \\ Department of Metal Physics, Ivan Franko National University, 8 Kyrylo and Mephodiy Str. 79005 Lviv, Ukraine \\ $\dagger$ Ural State Pedagogical University, 26 Cosmonavtov Av. 620017 Ekaterinburg, Russia \\ †nstitute of Metallurgy, Ural’s Division of Russian Academy of Sciences, 101 Amundsen Str. 620016 Ekaterinburg, Russia
}

MS received 5 January 2010; revised 6 July 2011

\begin{abstract}
Structure of $\mathrm{Bi}_{100-x} \mathrm{Ga}_{x}$ molten alloys containing 38.5, 50, 70 and 91.5 at. \% Ga has been studied by means of X-ray diffraction method and compared with viscosity measurements data. Significant changes in the structure factor profile were observed in vicinity of the concentration 70 at. \% Ga. The dynamic viscosity coefficient was calculated by use of a statistical atomic distribution model and a Born-Green kinetic theory. The concentration dependence of viscosity is in agreement with change of structure parameters obtained from diffraction data.
\end{abstract}

Keywords. Bi-Ga alloys; structure factor; X-ray diffraction method; viscosity; melts.

\section{Introduction}

The main features of Bi-Ga system are an absolute insolubility of components in a solid state and limited in liquid, that is pronounced in presence of wide concentration and temperature area of immiscibility in liquid one. Studies of such systems in liquid phase are important for understanding of miscibility gap formation mechanism on microscopic scale and relation of this mechanism with structure changes. During the last few years, most of the researchers have focused on the study of physical properties in the vicinity of critical point, where they reveal an anomalous behaviour. However, results of diffraction studies as well as physicochemical properties measurements are interpreted commonly separately of each other, because of limited availability of data, in which the relation between structure and properties is considered.

Constituents of this system, $\mathrm{Bi}$ and $\mathrm{Ga}$ being elements, their structures in liquid state have atomic distribution at which atomic volume decreases upon melting. $\mathrm{Ga}$ is more similar to typical metals in liquid state than $\mathrm{Bi}$. Particularly its coordination number is higher than for $\mathrm{Bi}\left(Z_{\mathrm{Ga}}=10.8 ; Z_{\mathrm{Bi}}=9.0\right)$, indicating larger fraction of covalent bonds in liquid $\mathrm{Bi}$ upon melting than in Ga. Between them exists the large difference in atomic size (29.6) whereas the electronegativity difference is small $(0 \cdot 15)$. Hence, the conditions for atomic segregation are satisfied.

Interest in $\mathrm{Ga}-\mathrm{Bi}$ system is also motivated by the fact that this is a unique system with large miscibility gap, whose both components are semimetals with above mentioned features

\footnotetext{
*Author for correspondence (yakymovych@online.ua)
}

in structure and properties upon melting. The other binary systems with miscibility gap in the liquid state are $\mathrm{Ga}-\mathrm{Pb}$, $\mathrm{Ga}-\mathrm{Tl}, \mathrm{Cu}-\mathrm{Pb}$, etc.

The structure and some kinetic properties (viscosity, diffusion) of liquid $\mathrm{Bi}-\mathrm{Ga}$ alloys were investigated earlier (Buhalenko et al 1992; Inui and Takeda 1993; Vollmann and Riedel 1996; Sklyarchuk et al 2008; Yagodin et al 2008). However, only a few of them are available, viz. the ones having relation between structure and kinetic properties for systems with a miscibility gap. In particular, the correlation between local structure and thermodynamic properties has been studied by Inui and Takeda (1993) by means of diffraction method, ultrasonic velocity and density measurements. Unfortunately, up to now no similar studies on correlation between structure and viscosity exist for Bi-Ga liquid alloys.

Thus, the main aim of this work was to find out the correlation between the viscosity concentration dependence behaviour and structure features in molten immiscible alloys, viz. in $\mathrm{Bi}-\mathrm{Ga}$ melts. For that reason, $\mathrm{X}$-ray diffraction (XRD) investigations were carried out for $\mathrm{Bi}_{100-x} \mathrm{Ga}_{x}$ liquid alloys $(x=8 \cdot 5,50,70,91.5$ at. \%) with subsequent calculation of viscosity coefficient using the statistical approach based on the formalism of Iida et al (1976) and the kinetic theory of Born and Green (1947). The calculated results of viscosity were compared with available literature data and our earlier published results (Sklyarchuk et al 2008).

\section{Experimental}

Ingots of bismuth and gallium (both of $99.99 \%$ purity) were used to prepare the alloy samples. 

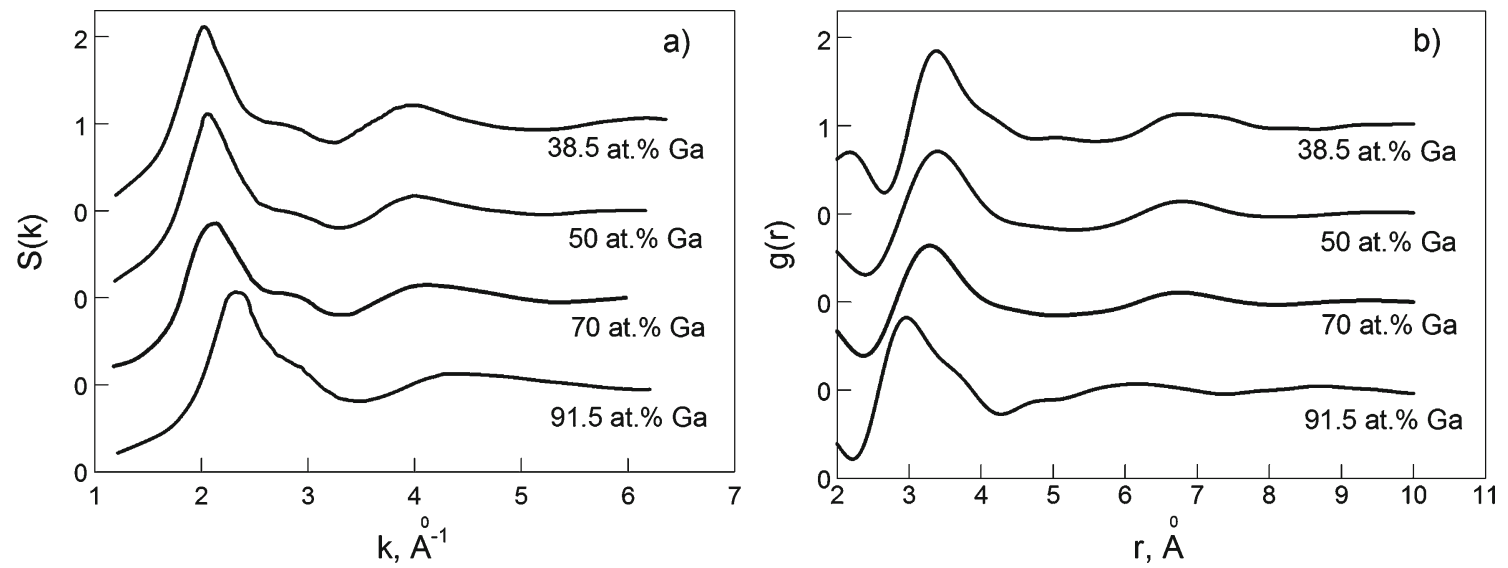

Figure 1. Structure factors (a) and pair correlation functions for Bi-Ga liquid alloys (b) at a temperature, $644 \mathrm{~K}$.

The XRD measurements were carried out by means of an X-ray diffractometer using $\mathrm{CuK} \alpha$ radiation monochromatized with $\mathrm{LiF}$ single crystal, in the $2 \theta$ range from $10^{\circ}$ up to $140^{\circ}$. A detailed description of the experimental technique was reported (Plevachuk et al 2009). The measuring of scattered intensity was done with an accuracy better than $2 \%$. Main structure parameters obtained from structure factor (SF) and pair correlation function (PCF) were analysed.

\section{Results and discussion}

Experimental SFs and PCFs for liquid $\mathrm{Bi}_{100-x} \mathrm{Ga}_{x}$ alloys of different Ga content are shown in figure 1. One can see that addition of $\mathrm{Ga}$ to $\mathrm{Bi}$ is accompanied with significant transformation of SFs. The change of their principal peak profile and shoulder on its right hand side is attributed to change in interatomic bounds which should cause the atomic redistribution. As it can be seen from this figure and table 1, the principal peak position in SF persists on addition of Ga up to 70 at. \%. Moreover, in case of alloys containing 38.5 and 50 at. $\%$ of gallium, parameter $k_{1}$ is even less than 1 for pure liquid $\mathrm{Bi}(2 \cdot 11 \AA$, table 1$)$. This parameter is the principal peak position in structure factor and is a characteristic of short range order in reciprocal space. Such behaviour has allowed us to suppose that the Bi-based structural units are not diluted by Ga-atoms. Most probably the Bi forms the self associated clusters. Tendency to segregation in atomic arrangement is also confirmed by the fact that shoulder is evidently pronounced in SF for each alloy, especially it is most pronounced for melt with 70 at. \% of Ga. Drastic change of SFs is observed for molten alloy, where content of $\mathrm{Ga}$ is 91.5 at.\%, revealing the transition to Bi-like structure.

Similar behaviour is also observed in concentration dependence of pair correlation functions (figure 1b). Significant increase of $\mathrm{Ga}$ concentration does not lead to corresponding decrease of most probable interatomic distance $r_{1}$ and one can see only a small reduction of this parameter. The second interatomic distance $r_{2}$ is also larger than in liquid $\mathrm{Bi}$.
This distance is somewhat less for alloy with 91.5 at.\% of Ga but it remains larger than corresponding value for liquid $\mathrm{Ga}$. Therefore, the anomalous behaviour of interatomic distances is observed not only in first coordination sphere, but also in the next spheres. It should be noted that the increase of interatomic distance is not accompanied by simultaneous decrease of atomic packing density, because the number of neighbours $(Z)$ is large for most investigated alloys (table 1). Results obtained from analysis of diffraction data are in accordance with the results of Inui and Takeda (1993), where the remarkable change in the structure factors have been observed at a critical concentration of around 70 at.\% $\mathrm{Ga}$. Attempt to remain at a large distance to neighbouring atoms is evidence of large concentration fluctuations in these molten alloys. Certainly, that such fluctuations should be displayed in viscosity coefficient values. Existence of remarkable concentration fluctuations is confirmed by results of diffraction studies by Buhalenko et al (1992), where dependence of structure factors on time at $T=573 \mathrm{~K}$ was investigated. It was found that atomic distribution significantly changes with time of holding at this temperature and the structure of melts is unchangeable when time of holding equals $2 \mathrm{~h}$ or more. Unfortunately the reason for such a behaviour is not analysed in this paper. From this work, existence of three concentration intervals for the structure is seen, but is not confirmed by our other studies.

Table 1. Structure parameters obtained from total structure factors and pair correlation functions.

\begin{tabular}{llllll}
\hline At\% Ga & $k_{1}\left(\AA^{-1}\right)$ & $k_{2}\left(\AA^{-1}\right)$ & $r_{1}(\AA)$ & $r_{2}(\AA)$ & $Z$ \\
\hline 91.5 & $2 \cdot 32$ & $4 \cdot 30$ & $2 \cdot 96$ & $6 \cdot 17$ & $8 \cdot 3$ \\
70 & $2 \cdot 14$ & $4 \cdot 10$ & $3 \cdot 30$ & $6 \cdot 76$ & $11 \cdot 7$ \\
50 & $2 \cdot 05$ & $4 \cdot 00$ & 3.40 & $6 \cdot 79$ & $10 \cdot 9$ \\
38.5 & $2 \cdot 03$ & 3.95 & $3 \cdot 38$ & $6 \cdot 81$ & $9 \cdot 2$ \\
Bi & $2 \cdot 11$ & $4 \cdot 12$ & $3 \cdot 38$ & $6 \cdot 60$ & $8 \cdot 8$ \\
Ga & $2 \cdot 52$ & 4.90 & $2 \cdot 82$ & $5 \cdot 6$ & $10 \cdot 4$ \\
\hline
\end{tabular}


In order to understand the features of atomic distribution in these alloys it is also interesting to reveal the correlation between the data observed by means of diffraction method and viscosity results, which are highly sensitive to structure changes. For that reason, we have calculated the viscosity data using the statistical approach which is a most simple approach and the kinetic theory of Born and Green (1947). The last of them was by using the diffraction data and potential of pair interaction.

At first, the viscosity of binary ideal solution $\eta^{\text {id }}$ was calculated according to additive approach:

$$
\eta^{\mathrm{id}}=\eta_{1} x_{1}+\eta_{2} x_{2}
$$

where $\eta_{1}, \eta_{2}, x_{1}, x_{2}$ are viscosity coefficients and atomic fractions of pure elements, respectively.

The second step is to account for a nonideal contribution to viscosity:

$$
\eta=\eta^{\mathrm{id}}+\Delta \eta
$$

where $\Delta \eta$ is the excess viscosity.

In order to calculate the excess viscosity we used the modified equation, which follows from the formalism of Iida et al (Terzieff 2008)

$$
\Delta \eta=\Delta \eta_{\sigma}+\Delta \eta_{\mathrm{M}}+\Delta \eta_{\mathrm{S}}+\Delta \eta_{\mathrm{V}}
$$

where $\Delta \eta_{\sigma}$ is the hard sphere part contribution to viscosity, which is the function of diameters difference, $\Delta \eta_{\mathrm{M}}$ the hard sphere part contribution to viscosity, which depends on nuclear weights difference of components; $\Delta \eta_{\mathrm{s}}$ the soft sphere part of viscosity, which is determined by an attractive part in interatomic potential, and $\Delta \eta_{\mathrm{V}}$ the additional compo- nent of viscosity, which is the dimensionless volume factor. The last equation can be written in following way:

$$
\begin{aligned}
\Delta \eta= & \left(x_{1} \eta_{1}+x_{2} \eta_{2}\right)^{*} \\
& \left(\begin{array}{l}
\alpha \frac{x_{1} x_{2}\left(\sigma_{1}-\sigma_{2}\right)^{2}}{x_{1} \sigma_{1}^{2}+x_{2} \sigma_{2}^{2}}+\beta\left[\left(1+\frac{x_{1} x_{2}\left(M_{1}^{1 / 2}-M_{2}^{1 / 2}\right)^{2}}{\left(x_{1} M_{1}^{1 / 2}+x_{2} M_{2}^{1 / 2}\right)^{2}}\right)^{1 / 2}-1\right] \\
+2 \gamma \Delta H /(R T)+2 \delta x_{1} x_{2}\left|V_{1}-V_{2}\right| /\left(V_{1}+V_{2}\right)
\end{array}\right),
\end{aligned}
$$

where $V_{1}, V_{2}, \sigma_{1}, \sigma_{2}, M_{1}, M_{2}$ are atomic volumes, hard sphere diameters and nuclear weights of components, respectively, $\Delta H$ the enthalpy of mixing. The fitting parameters $\alpha$, $\beta, \gamma, \delta$ are as follows: $\alpha=-4.05, \beta=12 \cdot 0, \gamma=-0.115$, $\delta=-2 \cdot 67$.

Atomic volume and hard sphere diameter data were calculated using the density (Yagodin et al 2008) and packing fraction values (Ostrovskii et al 1988). Enthalpy of mixing values was taken from Huber et al (2008).

Born and Green (1947) derived a formula for viscosity of a liquid in frame of classic kinetic theory:

$$
\eta=\frac{2 \pi}{15 V^{2}}\left(\frac{M}{k T}\right)^{1 / 2} \int_{0}^{\infty} g(r) \frac{\mathrm{d} \varphi(r)}{\mathrm{d} r} r^{4} \mathrm{~d} r,
$$

where $V$ is the nuclear volume of liquid; $M$ the nuclear weights of liquid; $k$ the Bolzmann's constant; $T$ the temperature; $g(r)$ the pair correlation function and $\varphi(r)$ the pair potential. The integral in (5) cannot be evaluated up to infinity limit, because we have the limited $k$-range of the experimental structure factors. On that reason some errors, which are known as cut-off errors are observed in $g(r)$. In order

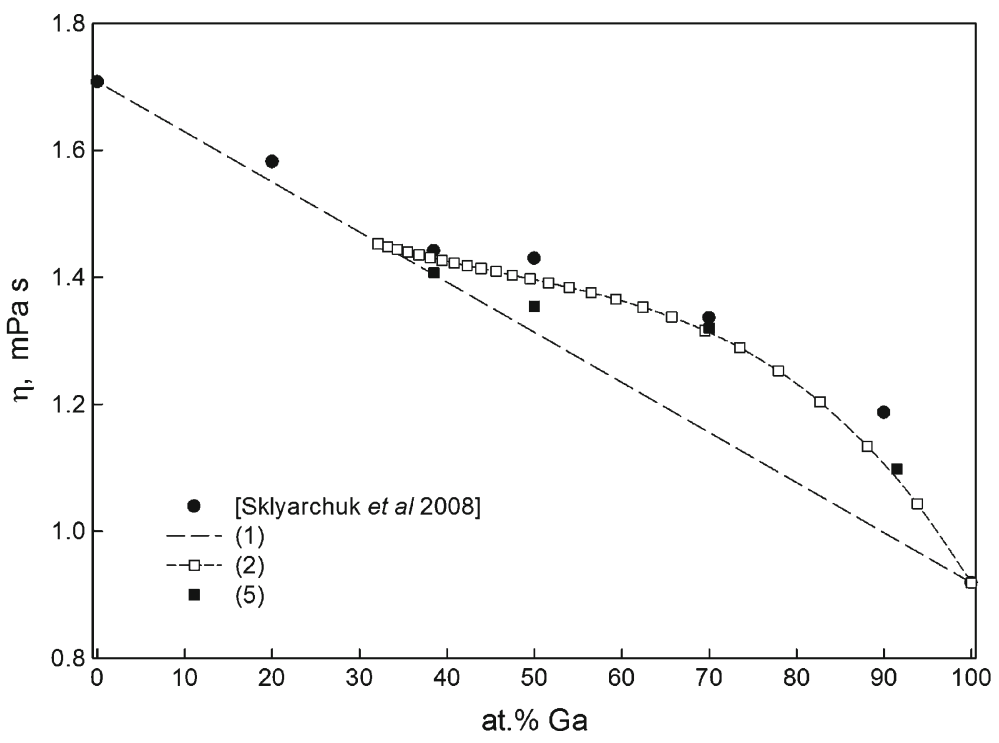

Figure 2. Concentration dependence of viscosity for Bi-Ga liquid alloys at a temperature of $644 \mathrm{~K}$. 


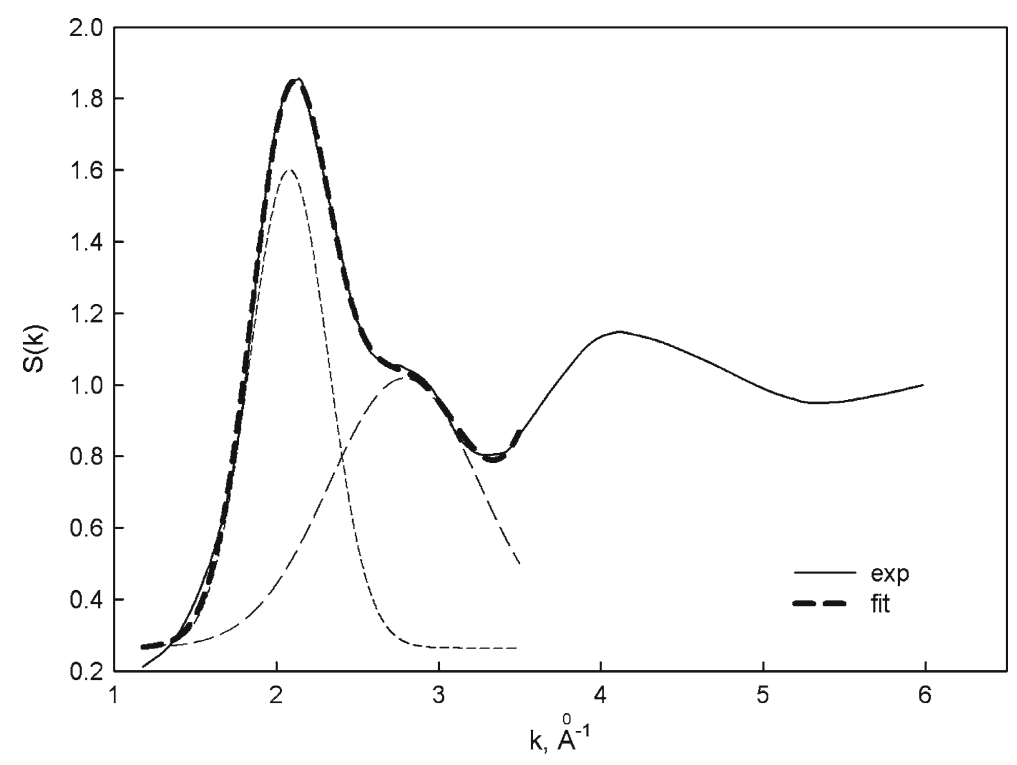

Figure 3. Computer fitting of principal peak for $\mathrm{Bi}_{30} \mathrm{Ga}_{70}$ liquid alloy.

to account for this error we have used special procedure (Kaplow et al 1965). In case of this model, we used the soft-sphere pair potential (Zelener et al 1981):

$$
\varphi(r)=k T \ln \left(1-\exp \left(-r^{2} / \sigma^{2}\right) .\right.
$$

By calculating the hard sphere diameters $\sigma$ of alloys, the packing fraction value was found to be 0.53 .

Results of viscosity calculation for above mentioned three models are shown in figure 2 . One can see that experimental viscosity is in agreement with calculated ones by (2) and (5), and deviates from data obtained by means of (1). Thus, here we have also confirmation about deviation of atomic arrangement from random distribution that correlates with results of $\mathrm{X}$-ray diffraction investigation. It can also be seen that the results obtained using (1), (2) and (5), respectively agree well with the experimental data in Bi-rich concentration region. The maximum deviation occurs in the interval from 50 at. $\%$ to 90 at. \% of $\mathrm{Ga}$ which is also in agreement with the diffraction results. Therefore, significant viscosity increase within interval, corresponding to miscibility gap is supposed to be related with large size of $\mathrm{Ga}-\mathrm{Ga}$ and $\mathrm{Bi}-\mathrm{Bi}$ structural units.

Compared to earlier published works we focus more on the shape of principal peak. Taking into account the asymmetric profile of principal peaks in SFs for liquid alloys as well as for $\mathrm{Ga}$ and $\mathrm{Bi}$ we carried out computer fitting of these maxima in additive assumption. The result of such fitting for molten alloy, containing 70 at. \% of $\mathrm{Ga}$ is shown in figure 3. As it can be seen from figure 3, the principal peak resulted from two kinds of structures: $\mathrm{Bi}$ - and Ga-like clusters (Wilson 1972).

We supposed that first of them corresponds to metallic clusters and second one to covalent clusters. Such parame- ters as peak positions and half height width have been determined from these partial maxima. The last of them was used for estimation of cluster size. It was found that metallic clusters for liquid $\mathrm{Ga}$ have a larger size than the ones for liquid Bi. Similar feature occurs for covalent clusters. On the other hand the parameters of both partial peaks are close to ones of liquid $\mathrm{Ga}$ within concentration interval from 0 to 50 at. $\mathrm{Bi}$ (near critical point) and then with the following Bi-content increasing, becomes more close to related parameters of liquid $\mathrm{Bi}$. Therefore, one can note that observed behaviour of structure parameters as well as viscosity data at concentrations, corresponding to miscibility gap, is in correlation with less size of clusters in Bi-enriched melts. It should be noted, that results of structure analysis are based not only on analysis of typical parameters obtained from SF and PCF, but also by more detailed analysis of principal peak shape in SF.

\section{Conclusions}

Structure of liquid $\mathrm{Bi}_{100-x} \mathrm{Ga}_{x}$ alloys reveals the tendency of formation of self-associated atomic groups (clusters), especially at the concentrations, corresponding to miscibility gap. Shape of principal peak is analysed in more detail that confirmed the cluster structure in liquid state. The viscosity data, calculated using simple models, also indicate the specific behaviour within this concentration region. Use of diffraction data in order to calculate the viscosity coefficient yields good agreement between calculated and experimental data. Conclusions about tendency to self-association derived from diffraction data are in correlation with ones obtained from viscosity calculations. 


\section{Acknowledgement}

This work was supported by a grant from the Fundamental Researches State Fund of Ukraine (No. F 28/329-2009).

\section{References}

Born M and Green H S 1947 Proc. R. Soc. A190 455

Buhalenko V V, Il'inskii A G, Romanov A V, Slusarenko S I and Chen S-Sh 1992 Metallofizika 1442 (in Russian)

Huber B, Richter K W, Flandorfer H, Mikula A and Ipser H 2008 Int. J. Mater. Res. 9918

Iida T, Ueda M and Morita Z 1976 Tetsu-to-Hagane 621169

Inui M and Takeda S 1993 J. Non-Cryst. Solids 156-158 153

Kaplow R, Strong S L and Averbach B L 1965 Phys. Rev. A138 1336
Ostrovskii O I, Grigoryan V A and Vishkarev A F 1988 Svoistva metallicheskikh rasplavov (Moscow: Metallurgiya) (in Russian)

Plevachuk Yu, Sklyarchuk V, Yakymovych A and Shtablavyi I 2009 Proceedings of the 6th international conference on electromagnetic processing of materials (EPM 2009) (Dresden: Forschungszentrum Dresden-Rossendorf) p. 415

Sklyarchuk V, Mudry S and Yakymovych A 2008 J. Phys.: Conf. Series 98062021

Terzieff P 2008 J. Alloys Compds. $\mathbf{4 5 3} 233$

Vollmann J and Riedel D 1996 J. Phys.: Condens. Matter 86175

Wilson J R 1972 Structure of liquid metals and alloys (Moscow: Metallurgiya) [Russian translation]

Yagodin D A, Filippov V V, Popel P S, Sidorov V E and Son L D 2008 J. Phys.: Conf. Series 98062019

Zelener B V, Norman G E and Filinov V S 1981 Perturbation theory and pseudopotential in statistical thermodynamics (Moscow: Nauka) 